\title{
NOTA
}

\section{Thlastocoris laetus MAYR, 1866 (HEMIPTERA: COREIDAE: ACANTHOCERINI) NUEVA PLAGA DE LA PIÑA.}

\author{
G. COUTURIER \\ ORSTOM/Museum National d'Histoire Naturelle 45, Rue Buffon, 75005 - Paris - France. \\ H. BRAILOVSKY \\ Departamento de Zoologia, Instituto de Biologia, UNAM, Apdo. Postal \# 70153 Mexico 04510 D.F. \\ R.A. ZUCCHI \\ Departamento de Entomologia - ESALQ/USP - C.P. 9, 13418-900 - Piracicaba,SP.
}

RESUMEN: Thlastocoris laetus (Hemiptera: Coreidae) es por primera vez señalado como plaga de la piña para la Amazonia brasileña y peruana comentando los daños que ocasiona. Se presenta la caracterización morfologica de esta especie, comparandola con Lybindus dichrous que también es reconocido como plaga de la piña en el Brasil. La distribución geográfica de ambas especies es citada.

Palabras clave: chinche, piña, Amazonia, Brasil, Perú, plaga

\section{Thlastocoris laetus MAYR, 1866 (HEMIPTERA: COREIDAE: ACANTHOCERINI), NOVA PRAGA DO ABACAXI.}

RESUMO: Thlastocoris laetus (Hemiptera: Coreidae) é assinalado pela primeira vez como praga do abacaxi na Amazônia brasileira e peruana. Os prejuízos ocasionados são comentados. $\hat{\mathbf{E}}$ apresentada a caracterização morfologica dessa espécie comparando-a com Lybindus dichrous, conhecida praga do abacaxi no Brasil. A distribuição geográfica dessas duas espécies é apresentada.

Descritores: percevejo, abacaxi, Amazônia, Brasil, Peru, pragas

\section{Thlastocoris laetus MAYR, 1866 (IEMIPTERA: COREIDAE: ACANTIIOCERINI), A NEW PINEAPPLE PEST.}

ABSTRACT: Thlastocoris laetus (Hemiptera: Coreidae) is reported for the first time as a pest of pineapple in Brazilian and Peruvian Amazon forest. The damages are commented. The species is characterized and compared with Lybindus dichrous, another coreidbug pest of pineapple in Brazil. The geographical distribution of the two species is reported.

Key wonds: bug, pineapple, pest, Amazonia, Brazil, Peru

\section{INTRODUCCION}

Desde hace varios años, se está realizando un inventario de los insectos plagas de los cultivos en la Amazonia brasileña (Manaus, $3^{0} 8^{\prime} \mathrm{S}, 60^{\circ} 1^{\prime} \mathrm{O}$ ) y peruana (Iquitos $3^{0} 45^{\prime} \mathrm{S}, 73^{\circ} 15^{\prime} \mathrm{O}$ y Jenaro Herrera $4^{0} 55^{\prime} \mathrm{S}, 73^{\circ} 40^{\prime} \mathrm{O}$ ). En estas tres localidades, se ha comprobado la presencia de Thlastocoris laetus Mayr, 1866 (Coreidae: Acanthocerini). BRAILOVSKY (1990) basándose en el material recolectado por Couturier y proveniente de Manaus (1986) y Jenaro Herrera
(1987) señala a la piña Ananas comosus (L) Merril (Bromeliaceae) como su posible planta hospedera.

Observaciones repetidas en los campos piñeros de la Amazonia brasileña y peruana, asi como en los cultivos experimentales del Instituto Nacional de Pesquisas de Amazonia en Manaus (INPA), en el Instituto Nacional de Insvestigación Agraria y Agroindustrial de Iquitos (INIAA) y en el Instituto de Investigación de la Amazonia Peruana en Jenaro Herrera (IIAP) nos permite confirmar que la piña es un hospedero importante de $T$. laetus el cual ocasiona daño a la misma. 


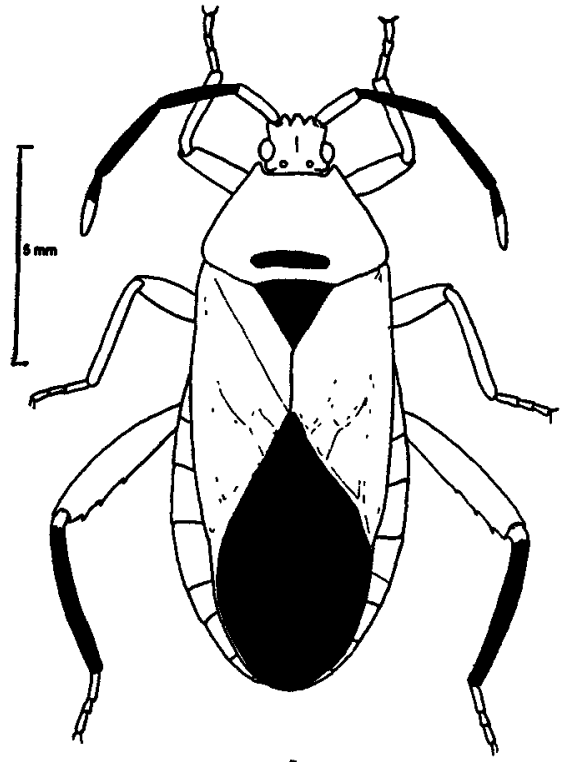

A

Figura 1a - Vista dorsal de Thlastocoris laetus Mayr.

Fueron estudiados especimenes de las colecciones del CIIF (Centro de Identificação de Insetos Fitófagos, Univ. Paraná, Curitiba), ESALQ (Escola Superior de Agricultura "Luiz de Queiroz", Univ. São Paulo, Piracicaba), Museu Nacional Rio de Janeiro. Los especimenes de $T$. laetus colectados en el marco del estudio fueron depositados en las colecciones citadas mas arriba y en las colecciones de la UNALM (Universidad Nacional Agraria La Molina, Lima, Perú), del INPA y del ORSTOM (Institut Français de Recherche Scientifique pour le Développement en Coopération, Paris, France).

\section{DAÑOS Y ASPECTOS BIOLOGICOS}

Los huevos son depositados en grupos de 203 o bien aislados, e irregularmente distribuídos en el pedúnculo del fruto, o en el propio fruto cuando éste mide entre 5 y 7 centimetros y eventualmente en las hojas del cogollo. Los huevos, las ninfas y los adultos siempre son encontrados de manera gregaria en la piña. Cuando en la misma planta se encuentran más de 12 a 15 ninfas así como adultos, el fruto no se desarrolla, las hojas sufren amarillamiento, secándose poco a poco y la planta muere.

Sci. agric., Piracicaba, 50(3):517-520, out./dez., 1993

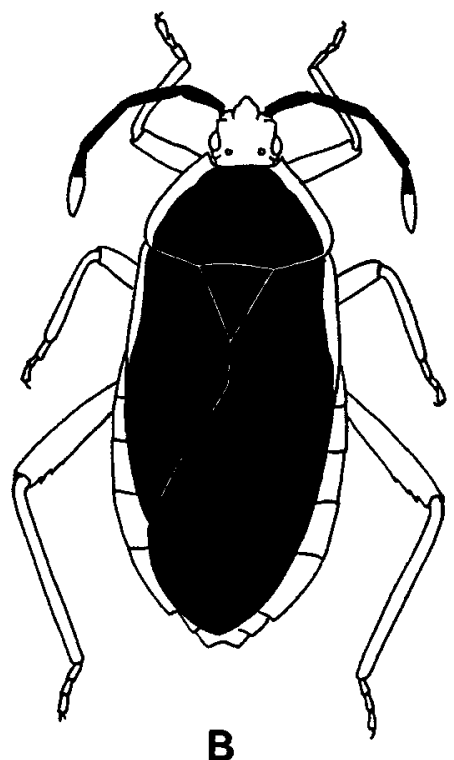

Figura $1 \mathrm{~b}$ - Vista dorsal de Lybindus dichrous Stal.
Caracteristicas diferenciales entre Thlastocoris laetus y Lybindus dichrous.

Estudios taxonómicos sobre la tribu Acanthocerini y el género Lybindus fueron realizados por O'SHEA (1980) y BRAILOVSKY (1984) respectivamente. Recientemente, BRAILOVSKY (1989) transfirio $L$. dichrous para Acanthocerini. Esta especie fue registrada por MARICONI (1953) como plaga de piña en el Brasil y tanto su comportamiento como los daños que causa en la planta hospedera son similares a los de $T$. laetus.

L. dichrous tiene el pronoto, el clavus y el corium de color negro y sólo los márgenes laterales y los ángulos humerales del pronoto así como gran parte del margen costal del corium de color anaranjado brillante; los segmentos dorsales del abdomen II al VI son amarillo anaranjado brillante, el I es negro y el VII bicolor combinando tonalidades anaranjadas con negro intenso; y el tylus está proyectado al frente (Fig. 1b). En T. laetus el pronoto es anaranjado brillante y con una banda transversal negra localizada en el tercio medio del margen posterior; el clavus, el corium y los segmentos dorsales del abdomen I a IV son enteramente de color amarillo anaranjado brillante, 


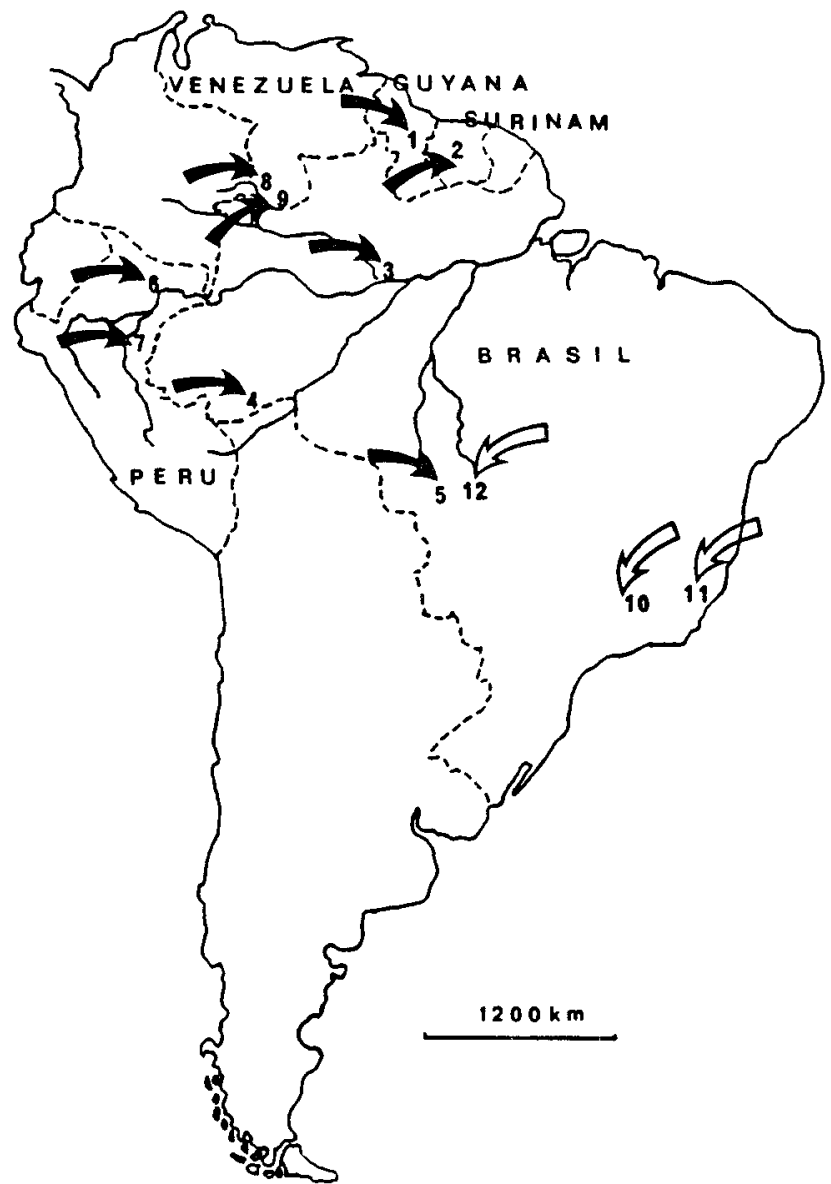

Figura 2 - Distribución geográfica de Thlastocoris laetus Mayr y de Lybindus dichrous Stal. Thlastocoris laetus (flecha negra) en Guyana: (1) y Surinam: (2) sin loca-lización precisa (Mayr, 1866, O'Shea, 1980, in Brailovsky 1990); en Brasil: (3) Manaus y alrededores (colectas G. Couturier y Museu Nacional - RJ) (4) Rio Branco (colección CIIF) (5) Cuiabá, Chapada dos Guimarães (colección CIIF); en Perú: (6) Iquitos y alrededo-res (7) Jenaro Herrera (Brailovsky, 1990, y colectas de G. Couturier y H. Inga); en Venezuela: (8) San Carlos de Rio Negro y (9) San Simon de Cucuy (Brailovsky, 1990). Lybindus dichrous (flecha blanca) en Brasil: (10) diversas localidades en el Estado de São Paulo (Mariconi, 1953), (11) Santa Teresa, Estado de Espírito Santo (colección CIIF), (12) Cuiabá (Mariconi, 1953; Brailovsky, 1984).

en tanto que los segmentos dorsales del abdomen $\mathrm{V}$ a VII son negros; el tylus está conspicuamente en declive (Fig. 1a).

El artejo antenal I varía conspicuamente en coloración a través de la serie revisada de ambos taxa, así en algunos ejemplares es amarillo anaranjado brillante, o bien pardo rojizo con reflejos anaranjados o combinación de ambos.

Sci. agric., Piracicaba, 50(3):517-520, out./dez., 1993

\section{DISTRIBUCION}

El ámbito distribucional de $T$. laetus parece limitado a la región Amazónica (Figura 2), tal como fue definida por PRANCE (1977), con excepción de Chapada de Guimarães, cerca de Cuiabá (Estado de Mato Grosso) localizada más al sur. Esta especie se distribuye en Venezuela, Guyana, Surinam, Brasil y Perú (BRAILOVSKY, 
1990). L. dichrous sólo es conocida para el Brasil, registrándosele para los Estados de São Paulo, Mato Grosso (MARICONI, 1953) y Espírito Santo (nuevo recorde).

\section{CONSIDERACIONES FINALES}

$T$. laetus al igual que $L$. dichrous causa daños severos en la piña y su control a largo plazo dependerá del conocimiento de su ciclo biológico, de la ecología del grupo y de sus posibles enemigos naturales, aspectos que están en estudio. Con respecto a $L$. dichrous, MARICONI (1953) sugiere un control preventivo destruyendo los cultivos abandonados y un control directo a través de la aplicación de insecticidas.

\section{REFERENCIAS BIBLIOGRAFICAS}

BRAILOVSKY, H. Notas acerca del género Lybindus Stal y descripción de una especie nueva (HemipteraHeteroptera-Coreidae-Coreini). Anales Instituto de Biolgía. Universidade Nacional Autónoma de México, Série Zoologia, México, v.55, n.1, p.155160,1984

BRAILOVSKY, H. Nuevos arreglos tribales dentro de la Familia Coreidae y descripcion de dos especies nuevas sudamericanas (Hemiptera-Heteroptera). Anales Instituto de Biolgía. Universidade Nacional Autónoma de México, Série Zoologia, México, v.59, n.2, p.159-180, 1989.
BRAILOVSKY, H. Géneros nuevos y especies nuevas de Coreidos Neotropicales (Hemiptera-HeteropteraCoreidae: Acanthocerini, Leptoscelidini y Anisoscelidini). Anales Instituto de Biolgía. Universidade Nacional Autónoma de México, Série Zoologia, México, v.61, n.1, p.107-123, 1990.

MARICONI, F.A.M. O percevejo do abacaxi (Lybindus dichrous Stal, 1859). O Biológico, São Paulo, v.19, n.9, p.155-162, 1953.

O'SHEA, R. A generic revision of the Acanthocerini (Hemiptera: Coreidae: Coreinae). Studies on Neotropical Fauna and Environment, Amsterdan, v.15, p.57-80, 1980 .

PRANCE, G.T. The phytogeographic subdivisions of Amazonia and their influence on the selection of biological reserves. In: PRANCE, G.T., ELIAS, T.S., (Ed.). Extinction is forever. New York: The New York Botanical Garden, 1977. p.195-213.

Enviado para publicação em 26.07 .93

Aceito para publicação em 23.08 .93 